07,11

\title{
Резонансы пространственного распределения дислокаций при взаимодействии со структурным параметром порядка
}

\author{
() Ю.Д. Заворотнев ${ }^{1}$, А.Ю. Захаров ${ }^{2}$, Л.С. Метлов ${ }^{1}$ \\ ${ }^{1}$ Донецкий фризико-технический институт им. А.А. Галкина, \\ Донецк, Украина \\ ${ }^{2}$ Новгородский государственный университет им. Ярослава Мудрого, \\ Великий Новгород, Россия \\ E-mail: zavorotnev.yurii@mail.ru
}

(Поступила в Редакцию 9 января 2018 г.)

Изучено взаимодействие структурной подсистемы с дислокациями при наложении упругой деформации кручения и дополнительного одноосного пространственно неоднородного и постоянного во времени давления. Рассмотрение проведено в рамках феноменологической теории Ландау при отказе от приближения постоянства модулей неприводимых векторов. Показано появление в таких условиях пространственных резонансов плотности дислокаций.

Один из авторов (А.Ю. Захаров) признателен Министерству образования и науки РФ за финансовую поддержку в рамках проектной части госзадания (грант 3.3572.2017/ПЧ).

DOI: 10.21883 /FTT.2018.07.46121.001

\section{1. Введение}

При изучении свойств твердых тел применяются различные внешние воздействия. В этом случае система перестраивается под действием каких-либо нетепловых параметров и могут произойти изменения некоторых характеристик кристалла в результате квантового перехода. Кристаллические параметры можно разбить на две группы. К первой принадлежат параметры непосредственно зависящие от воздействия, ко второй не зависящие. В силу наличия различного рода взаимодействий изменения в одной группе параметров влекут за собой изменения в другой. Одним из эффективных внешних воздействий является кручение кристалла вдоль некоторой оси. В работе [1] представлен хороший обзор результатов, полученных при применении этой методики.

Необходимо отметить, что указанным методом можно изучать эффекты, обусловленные достаточно малыми взаимодействиями. В частности, в работах [2-7] рассмотрено взаимодействие структурной и магнитной подсистем при отказе от предположения постоянства величины модулей соответствующих параметров порядка (ПП). Показано, что при наложении на идеальный кристалл интенсивной пластической деформации кручением (ИПДКР) на начальной упругой стадии возникают пространственные амплитудные и частотные модуляции этих ПП и можно создавать различные их пространственные распределения. Оказалось также, что при дополнительном наложении одноосного пространственно неоднородного и постоянного во времени давления (ПОД) могут возникнуть резонансные эффекты [7].
Реальные кристаллы имеют различного рода дефекты. Эволюция плотности структурных дефектов при ИПДКР может быть описана в расках неравновесной эволюционной термодинамики $[8,9]$. В работе [10] авторами был предложен вариант теории, сочетающий классическую теорию фазовых переходов для описания эволюции структурного параметра порядка и неравновесную эволюционную термодинамику, описывающую соподчиненную параметру порядка эволюцию структурных дефектов. В рамках этой теории структурные дефекты взаимодействуют со структурным параметром порядка и поэтому их плотность может пространственно меняться. Было показано, что при наложении упругой деформации кручения (УДК) и определенных условиях в кристалле может возникнуть сверхрешетка дислокаций. Настоящая работа является продолжением публикации [10] и отличается от нее тем, что изучаются эффекты, возникающие при дополнительном приложении к кристаллу одноосного пространственно неоднородного и постоянного во времени давления. Суммарное приложение УДК и ПОД приводит к появлению пространственных резонансных явлений.

\section{2. Теория}

Рассмотрим модельный кристалл с дислокациями. Пусть при понижении температуры произошел некоторый структурный фазовый переход (ФП), который может быть как первого, так и второго рода, и кристалл находится в устойчивой фазе. Эта фаза характеризуется структурным параметром порядка (ПП), который является линейной комбинацией отклонений ионов эле- 
ментарной ячейки, т.е. является векторной величиной. Этот ПП преобразуется по соответствующему неприводимому представлению группы симметрии кристалла. Предположим, что симметрия образовавшейся структуры допускает существование инвариантов Лифшица, однако в силу некоторых причин длиннопериодическая геликоидальная структура не возникает. Так же как и в работе [10], будем описывать дислокации с помощью их плотности (дислокационный ПП) [9]. Соответствующий ПП в силу своего определения преобразуется по полносимметричному неприводимому представлению группы симметрии кристалла.

В силу наличия взаимодействия между структурным ПП и дислокациями изменение первого при наложении некоторого внешнего воздействия должно оказать влияние и на плотность дислокаций. В работе [10] рассмотрено поведение обоих ПП вблизи и вдали от вышеуказанного ФП при наложении упругой деформации кристалла (УДК) в виде винтового крутящего момента с вектором распространения вдоль оси $O Z$. При таком воздействии возникает пространственная периодическая спиральная структура, т. е. фактически имеет место ФП второго рода, происходящий без участия температурных флуктуаций. Поставленная задача была сведена к изучению взаимодействия между дефектным и структурным ПП. Теперь предположим, что кроме УДК к кристаллу приложено пространственно синусоидальное и постоянное во времени одноосное давление (ПОД) по оси $O Y$. Тогда, в рамках феноменологической теории Ландау плотность неравновесного термодинамического потенциала (НТДП) запишется в виде

$$
\begin{aligned}
\Phi= & \frac{\beta_{1}}{2} q^{2}+\frac{\beta_{2}}{4} q^{4}+\frac{\beta_{3}}{6} q^{6}-\gamma_{1} M^{r}\left(q_{x} \frac{\partial y}{\partial z}-q_{y} \frac{\partial q_{x}}{\partial z}\right) \\
& +\gamma_{2} M^{s}\left(\left(\frac{\partial q_{x}}{\partial z}\right)^{2}+\left(\frac{\partial q_{y}}{\partial z}\right)^{2}\right)+\gamma_{3} q^{2} h+\gamma_{4} q^{2} h^{2} \\
& +\gamma_{5}\left(\frac{\partial h}{\partial z}\right) \varphi_{1} h+\frac{\varphi_{2}}{2} h^{2}+\frac{\varphi_{3}}{3} h^{3}+\frac{\varphi^{4}}{4} h^{4} \\
& +\gamma_{6} P_{y} q_{y}\left(1+\gamma_{7} \cos \left(t^{*} z\right)\right)
\end{aligned}
$$

где $\beta_{i}, \gamma_{i}, \varphi_{i}$ - феноменологические коэффициенты; $h, q$ - дефектный и структурный ПП соответственно; $M$ - модуль крутящего момента; $r, s$ - показатели степеней, величина которых, согласно результатам работы [3], $r=6, s=2, P_{y}$ - модуль одноосного и постоянного во времени давления вдоль оси $O Y$. В нашей модели описание магнитной спиральной структуры [11] распространено на случай кристаллической структуры, возникающей при УДК. Слагаемые с пространственными производными описывают винтовую деформацию (несоразмерная длиннопериодическая спиральная структура) и содержат пропорциональный моменту множитель, т.е. при $|M|=0$ отсутствует пространственная спиральная структура.
В потенциале (1) следует учесть также упругое взаимодействие. Переменные, описывающие это взаимодействие, можно исключить, определяя с помощью уравнений состояний их равновесные значения в зависимости от величины структурного ПП. После подстановки найденных выражений в потенциал получаем новый НТДП. Он формально будет совпадать с НТДП без учета упругого взаимодействия. При этом новые константы будут зависеть от температуры и давления. В дальнейшем будем предполагать, что процедура исключения проведена. При отказе от предположения постоянства модулей неприводимых векторов в общем случае неоднородного распределения дислокаций получаем следующую систему уравнений Эйлера

$$
\left\{\begin{array}{l}
2 \gamma_{2} M^{s} \frac{\partial^{2} q_{x}}{\partial z^{2}}+\gamma_{1} M^{r} \frac{\partial q_{y}}{\partial z}-q_{x}\left(\beta_{1}+\beta_{2} q^{2}\right. \\
\left.\quad+\beta_{3} q^{4}+2 \gamma_{3} h+2 \gamma_{4} h^{2}\right)=0, \\
2 \gamma_{2} M^{s} \frac{\partial^{2} q_{y}}{\partial z^{2}}-\gamma_{1} M^{r} \frac{\partial q_{x}}{\partial z}+\gamma_{6} P_{y}\left(1+\gamma_{7} \cos \left(t^{*} z\right)\right) \\
\quad-q_{y}\left(\beta_{1}+\beta_{2} q^{2}+\beta_{3} q^{4}+2 \gamma_{3} h+2 \gamma_{4} h^{2}\right)=0, \\
2 \gamma_{s} \frac{\partial^{2} h}{\partial z^{2}}-\left(\varphi_{1}+\varphi_{2} h+\varphi_{3} h^{2}+\varphi_{4} h^{3}+\gamma_{3} q^{2}+2 \gamma_{4} q^{2} h\right)=0 .
\end{array}\right.
$$

Эта система в общем случае состоит из трех дифференциальных уравнений. Если первоначальное распределение дислокаций однородно, то $\gamma_{5}=0$ и имеем смешанную систему из двух дифференциальных уравнений и одного алгебраического.

Систему (2) решить аналитически затруднительно. Поэтому с помощью математического пакета MathCad 15 был проведен численный анализ. При этом также был проведен Фурье-анализ полученных решений, который позволил выявить наличие кратных и некратных гармоник. Везде в дальнейшем будем предполагать, что $\varphi_{1}>0, \varphi_{2}<0, \varphi_{3}>0, \varphi_{4}, 0$.

\section{3. Обсуждение результатов}

Для облегчения сравнения результатов данной работы и [10] в настоящем разделе будем придерживаться такого же порядка рассмотрения, как и в [10]. Везде в дальнейшем будем учитывать, что $\beta_{1}<0, \beta_{2}>0$. Рассмотрим случай однородного распределения дислокаций (система (2)). Пусть $\gamma_{2}=0, \gamma_{4}<0$.

1) В этом случае имеет место структурный ФП второго рода. При отсутствии ПОД модуль структурного ПП изменяется по несинусоидальному периодическому закону. В результате при наложении ПОД в случаях совпадения максимумов и минимумов колебаний модуля дислокационного ПП и возмущающего давления возникает пространственный резонанс, проявляющийся в увеличении односторонних пространственных амплитудных колебаний структурного и дислокационного ПП в определенных ограниченных областях пространства (рис. 1). При этом не возникают бездислокационные зоны. 




Pис. 1. Сплошной, пунктирной и штриховой линиями представлены пространственные зависимости дислокационного ПП, структурного ПП и ПОД.



Рис. 2. Сплошной и пунктирной линиями представлены пространственные зависимости дислокационного и структурного ПП соответственно.

2) $\beta_{2}<0$. Система находится по температуре ниже ФП первого рода. При отсутствии ПОД возможно появление дислокационной сверхрешетки дислокаций [10]. Если приложить ПОД, то в этой решетке возникают пространственные биения (рис. 2) с пространственной амплитудной модуляцией, большей, чем в случае 1.

Рассмотрим случай однородного распределения дефектов с другим типом взаимодействия, т.е. $\gamma_{4}=0$, $\gamma_{2}<0$.

3) $\beta_{2}<0$. Такой знак коэффициента НТДП описывает ФП первого рода структурной подсистемы. При отсутствии ПОД также возникает сверхрешетка дислокаций, но ее структура отличается от случая 3. Наложение постоянного давления приводит к появлению пространственной частотной модуляции (рис. 3). При этом размеры областей с отрицательными значениями плотности уменьшаются и увеличиваются величины амплитуды колебаний плотности дислокаций. В случае $\gamma_{2}>0$ в дислокационной решетке возникают слабая амплитудная и сильная частотная пространственные модуляции (рис. 4).

4) $\beta_{2}>0$. Имеем ФП второго рода в структурной подсистеме. Противофазность сохраняется. При дости-

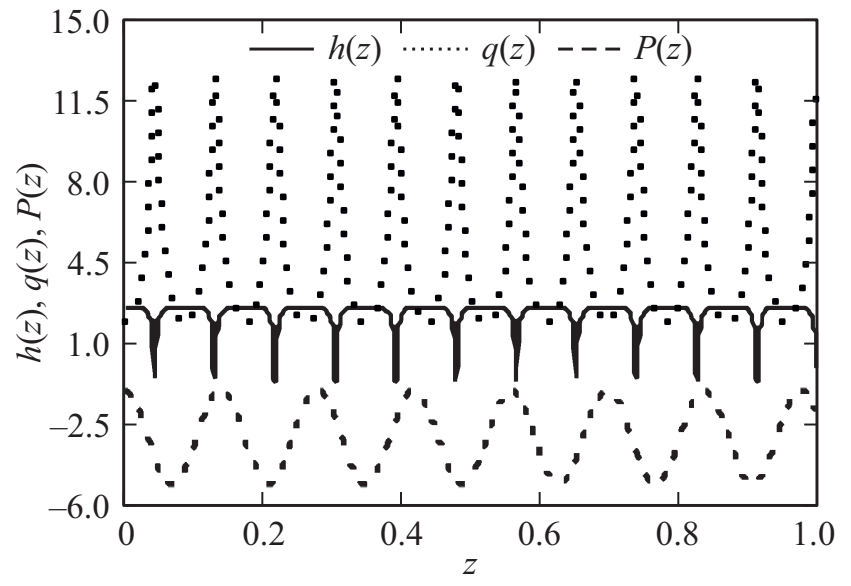

Рис. 3. Сплошной, пунктирной и штриховой линиями представлены пространственные зависимости дислокационного ПП, структурного ПП и ПОД.



Рис. 4. Сплошной, пунктирной и штриховой линиями представлены пространственные зависимости дислокационного ПП, структурного ПП и ПОД. График ПОД для наглядности сдвинут вниз.

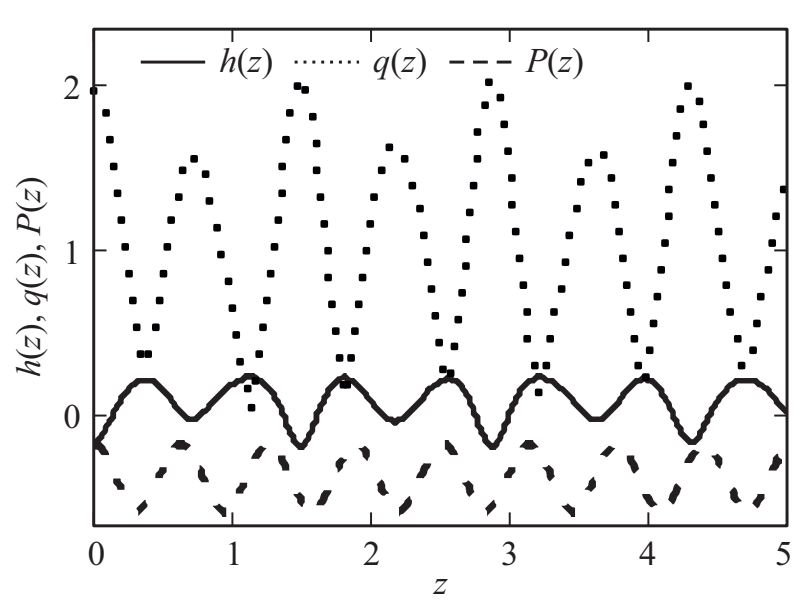

Рис. 5. Сплошной, пунктирной и штриховой линиями представлены пространственные зависимости дислокационного ПП, структурного ПП и ПОД. График ПОД для наглядности сдвинут вниз. 


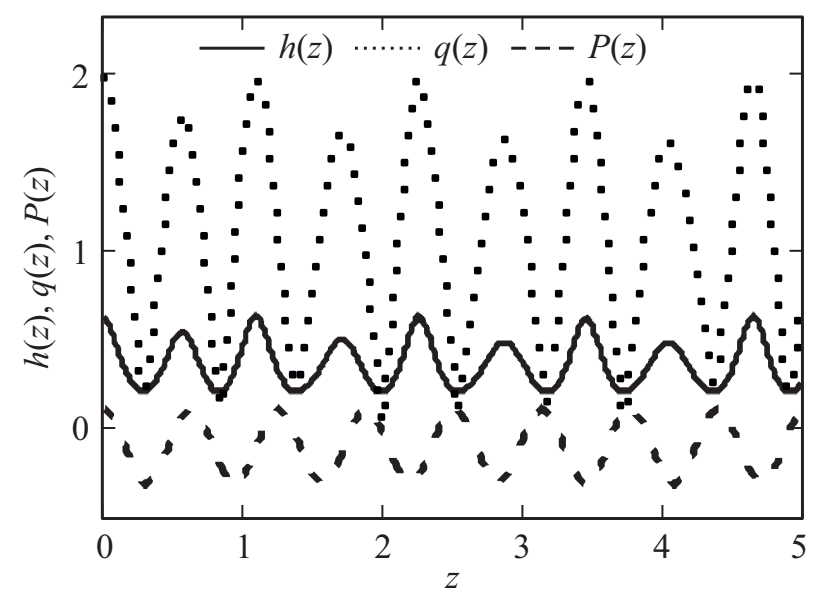

Pис. 6. Сплошной, пунктирной и штриховой представлены пространственные зависимости дислокационного ПП, структурного ПП и ПОД. График ПОД для наглядности сдвинут вниз.



Рис. 7. Сплошной, пунктирной и штриховой линиями представлены пространственные зависимости дислокационного ПП, структурного ПП и ПОД. График ПОД для наглядности сдвинут вниз.

жении резонанса появляются пространственные амплитудная и частотная модуляция обоих ПП. Это приводит появлению чередующихся бездислокационных областей разной длины (рис. 5). Анализ Фурье показал, что определяющими являются гармоники, расположенные в окрестностях 14-й и 28-й и ответственные за частотную модуляцию. Гармоники, которые сгруппированы в районах 40-й и 48-й, описывают размах амплитудной модуляции. При увеличении момента кручения возможно двукратное увеличение периода появления областей с идеальной решеткой. Если $\gamma_{2}>0$, также имеет место синфазность и появляется пространственная амплитудная модуляция обоих ПП (рис. 6).

Пусть в кристалле создано неоднородное распределение дислокаций, которое подчиняется синусоидальному закону, период которого сравним с периодом внешнего кручения (система уравнений $(2), \gamma_{5} \neq 0$ ).
5). $\beta_{2}<0, \gamma_{2}<0$. В структурной подсистеме - ФП первого рода. Синфазность при наложении ПОД сохраняется, структура бездислокационных областей не меняется (рис. 7), сохраняется синфазность пространственных колебаний и появляется амплитудная пространственная модуляция обоих ПП. При $\gamma_{2}>0$ сохраняется противофазность и в областях, близких к резонансу, на максимумах зависимости $h(z)$ наблюдаются особенности, при которых первая производная испытывает скачки первого рода. При этом амплитудная модуляция появляется только на графике структурного ПП (рис. 8 ).

6). $\beta_{2}>0, \gamma_{2}<0$. Имеем ФП второго рода. Наложение ПОД с периодом, примерно равным периоду пространственных колебаний дислокационного ПП, приводит к появлению длиннопериодической пространственной амплитудной модуляции (рис. 9). Верхняя и нижняя составляющие этой модуляции имеют пример-

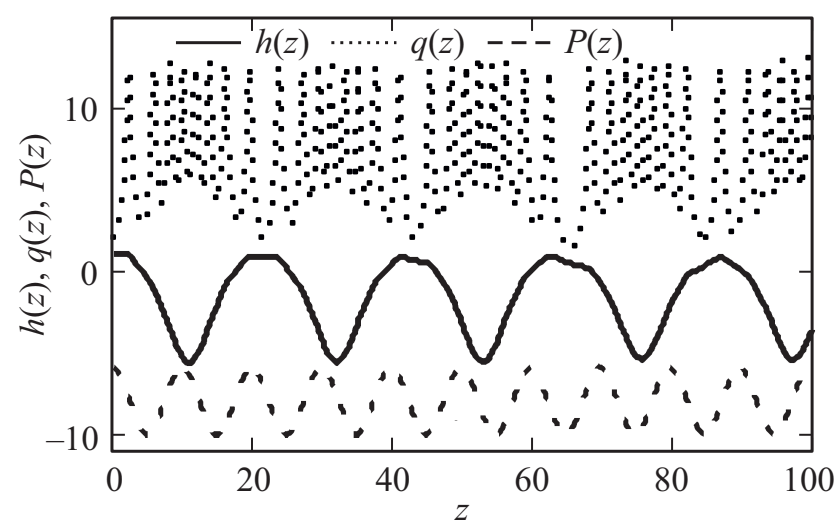

Рис. 8. Сплошной, пунктирной и штриховой линиями представлены пространственные зависимости дислокационного ПП, структурного ПП и ПОД. График ПОД для наглядности сдвинут вниз.

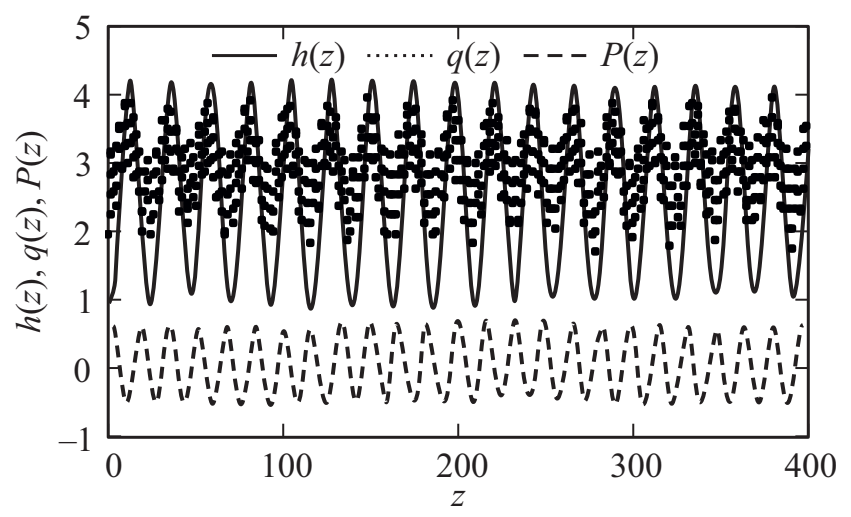

Рис. 9. Поведение структурного и дислокационного ПП при ФП первого рода при первоначальном синусоидальном распределении дислокаций. Сплошной, пунктирной и штриховой линиями представлены пространственные зависимости дислокационного ПП, структурного ПП и ПОД. График ПОД для наглядности сдвинут вниз. 


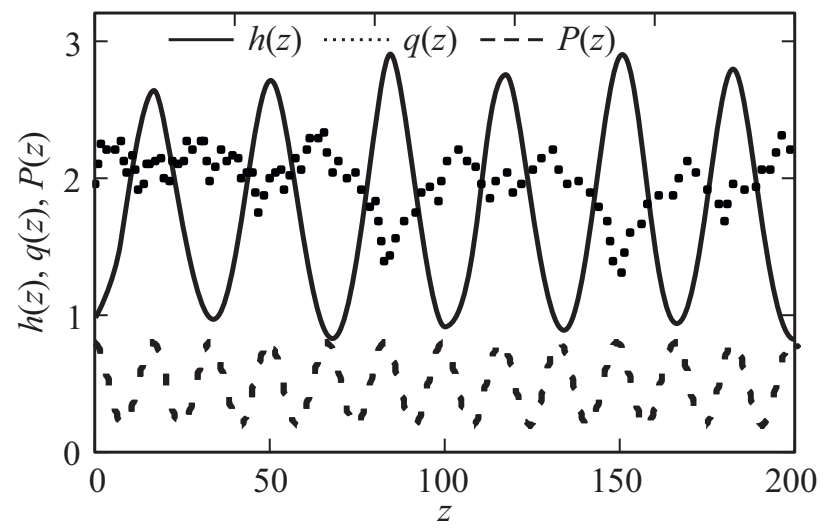

Рис. 10. Поведение структурного и дислокационного ПП при ФП второго рода и первоначальном синусоидальном распределении дислокаций. Сплошной, пунктирной и штриховой линиями представлены пространственные зависимости дислокационного ПП, структурного ПП и ПОД. График ПОД для наглядности сдвинут вниз.

но одинаковые амплитуды и находятся в противофазе. В случае $\gamma_{2}>0$ ПОД в резонансе оказывает более сильное влияние на структурный и дислокационный ПП (рис. 10). Пульсации амплитудной модуляции заметны на гораздо меньших расстояниях, чем в предыдущем тохслучае.

\section{4. Заключение}

1) Показана возможность изучения взаимодействия векторного (структурный ПП) и скалярного (плотность дислокаций) полей с использованием формализма Ландау при отказе от приближения постоянства модулей неприводимых векторов.

2) Оказалось, что результате совместного приложения к кристаллу упругой деформации кручения и одноосного пространственно неоднородного и постоянного во времени давления в дислокационной подсистеме могут возникать пространственные резонансы.

3) Путем варьирования величины вектора распространения пространственных колебаний неоднородного давления можно формировать различные распределения изменений модулей ПП.

\section{Список литературы}

[1] B.B. Straumal, A.R. Kilmametov, A. Korneva, A.A. Mazilkin, P.B. Straumal, P. Zieba, B. Baretzky. J. Alloys Comp. 707, 20 (2016).

[2] Ю.Д. Заворотнев. ФНТ 39, 178 (2013).

[3] Yu.D. Zavorotnev, E.H. Pashinska, V.N. Varyukhin, O.Yu. Popova. J. Magn. Magn. Mater. 349, 244 (2014).

[4] Ю.Д. Заворотнев, Е.Г. Пашинская, В.Н. Варюхин. Изв. РАН. Сер. физ. 78, 1011 (2014).
[5] Ю.Д. Заворотнев, Е.Г. Пашинская. ФНТ 40, 1237 (2014).

[6] Ю.Д. Заворотнев, Е.Г. Пашинская. ФТТ 58, 647 (2016).

[7] Yu.D. Zavorotnev, O.Yu. Popova, K.V. Gumennyk. J. Magn. Magn. Mater. 442, 391 (2017).

[8] L.S. Metlov. Phys. Rev. Lett. 106, 165506 (2011).

[9] L.S. Metlov. Phys. Rev. E 90, 022124 (2014).

[10] Ю.Д. Заворотнев, А.Ю. Захаров, Л.С. Метлов. ФТТ 59, 2266 (2017).

[11] И.Е. Дзялошинский. ЖЭТФ 46, 1420 (1964).

Редактор Ю.Э. Китаев 